\title{
Le développement thématique dans les pratiques d'écriture en
} contexte scolaire/académique

Maria Antónia Coutinho

\section{Citer ce document / Cite this document :}

Coutinho Maria Antónia. Le développement thématique dans les pratiques d'écriture en contexte scolaire/académique. In: La Lettre de I'AIRDF, n54, 2013. pp. 39-41;

doi : https://doi.org/10.3406/airdf.2013.1983

https://www.persee.fr/doc/airdf_1776-7784_2013_num_54_1_1983

Fichier pdf généré le 15/03/2019 


\section{LE DÉVELOPPEMENT THÉMATIQUE DANS LES PRATIQUES D'ÉCRITURE EN CONTEXTE SCOLAIRE/ACADÉMIQUE}

Comme on l'a fréquemment souligné, l'interactionnisme socio-discursif (dorénavant ISD) n'est pas une théorie (sociologique, psychologique ou linguistique) et il ne doit pas être confondu avec un modèle d'analyse de discours ou de textes. Il s'agit d'un cadre épistémologique (interactionniste social) et politique, qui met en évidence le rôle fondamental du langage dans la constitution de la personne consciente et dans son développement tout au long de la vie (Bronckart, 1997). Cela étant, l'analyse des textes et des discours ne constitue pas une finalité en elle-même, mais cela n'empêche pas les recherches menées dans le cadre de l'ISD de contribuer à l'évolution des connaissances sur le fonctionnement des uns et des autres, ni de profiter des données qui résultent de recherches plus strictement linguistiques.

C'est dans le cadre de l'ISD que se situe la présente contribution, où nous nous proposons de réfléchir à partir de la notion spécifique de figures d'action (Bronckart \& Bulea, 2006 ; Bulea, 2007), en essayant de comprendre dans quelle mesure l'émergence de figures peut être reliée à une description générale du fonctionnement des textes - envisagé du point de vue du développement de la personne (adulte) et, de façon particulière, du développement des performances scolaires/académiques.

Sans reprendre dans le détail l'approche que l'ISD fait des textes et des discours, rappelons que les textes constituent des correspondants empiriques des activités sociales (collectives) où ils prennent place. Ils relèvent toujours d'un genre, sélectionné dans le répertoire de genres associé à l'activité dont il est question, répertoire toujours plus ou moins flou, qui change et s'adapte mais qui constitue néanmoins un cadre de référence, pour une époque donnée. D'un autre côté, le fait que toute activité collective est assurée, dans le temps et dans l'espace (dans l'histoire), par des agents singuliers, implique que chaque texte correspond à une action particulière, c'est-à-dire à l'inscription-intervention de quelqu'un (ou d'un petit groupe) dans le cadre d'une activité collective donnée. Cette action dépend de la maîtrise, plus ou moins développée et consciente, du répertoire de genres associés à l'activité concernée, mais elle dépend aussi, de façon décisive, de la maitrise des types de discours et donc, nécessairement, des ressources de la langue naturelle en usage. Les textes étant donc des produits foncièrement sociaux, les types de discours et les formes et structures linguistiques qui les configurent constituent les solutions techniques de leur élaboration.

C'est par rapport à cette approche globale que la notion de figures d'action introduit de nouvelles interrogations. Identifiées et décrites d'après l'analyse d'entretiens dans un contexte de recherche précis, les figures d'action correspondent à des «produits interprétatifs » qui se mettent en place lorsque les types de discours organisent un contenu thématique de l'ordre de l'agir (Bulea, 2007, 2009). Par ailleurs, E. Bulea (2009 : 148-9) a bien souligné le caractère sémiologique des figures, qui préservent les propriétés générales des signes (caractère biface, nature psychique et différentielle) tandis que les propriétés caractéristiques des signes linguistiques (arbitrarité, linéarité et caractère discret) se trouvent modifiées. En ce qui concerne le caractère discret, Bulea a observé qu'il est affaibli dans les figures d'action, une fois que « leur début et leur fin sont gérés textuellement et non systémiquement » (Bulea, 2009 : 149).

Ce qui vient d'être très brièvement exposé soulève les questions qui sont au centre de la présente démarche. D'un côté, il s'agit de savoir si le façonnement discursif d'autres thèmes implique aussi l'émergence de figures - ou d'autres figures, plus ou moins proches des figures d'action déjà identifiées (action occurrence, action événement passé, action expérience, action canonique, action définition). Si l'extrême diversité de thèmes possibles peut nous faire douter du succès de l'entreprise, il faut en même temps admettre que la possibilité de rendre compte de la composante thématique ne peut se réduire à la description du thème de l'agir - pour important qu'il soit, par rapport aux enjeux de l'ISD. D'un autre côté, le fait que les figures 
d'action correspondent à des «macro-signes » (Bulea, 2009: 149) établis textuellement, comme nous l'avons déjà souligné, ne peut que renforcer le même questionnement : le contenu thématique de l'ordre de l'agir serait-il le seul à déclencher l'émergence de tels macro-signes ? Si ce n'est pas le cas, que peut-on en dire? Quelles régularités peut-on identifier, lorsque c'est d'autres thèmes qu'il s'agit ?

La réponse à ces questions nous conduirait à une compréhension plus fine de l'articulation entre organisation thématique et organisation discursive (conformément à la révision du modèle de l'architecture textuelle proposée par Bronckart, 2008). Mais elle devrait permettre aussi de mieux cerner le rapport entre genre de texte et texte empirique : si le premier se laisse décrire par un plan (plan conventionnel, selon les termes de Adam, 2011 : 205), la description du second ne peut dépasser le processus morphogénétique par lequel émergent ces entités (ou macro-signes) dont on pourra peut-être parler en termes de figures du thème.

Les données dont nous disposons pour le moment ne dépassent pas une étape exploratoire de la recherche. Elles nous permettront, en tout cas, de mieux décrire l'enjeu dont il est question. Nous avons donc travaillé sur trois ensembles de textes. Nous avons analysé un ensemble de dix interviews - en essayant de garder, du moins en partie, des conditions de comparaison par rapport aux entretiens analysées, lors de l'établissement des figures d'action. Il s'agit d'interviews publiées par des journaux et des revues spécialisées (sur le vin); l'interviewé était toujours le même, un chanteur portugais, et le motif de l'interview, son dernier CD-Rom qui venait de paraître au Portugal, à l'époque. Dans l'impossibilité de reproduire ici les textes (en portugais) et le détail de leur analyse, nous ne bornerons à dire qu'on y retrouve, en gros, les mêmes figures d'action que celles qui ont déjà identifiées (Bulea, 2007). La seule figure qui n'apparait pas est la figure d'action canonique - probablement, parce qu'il n'y a pas de textes prescriptifs (significatifs), en ce qui concerne ce dont il est question : le vin, la musique, l'activité créatrice d'un chanteur/auteur. Nous avons aussi observé les introductions des interviews - dans tous les cas où les textes (ou leurs plans) comprenaient une telle section, correspondant à la présentation que le journaliste fait du chanteur et/ou du prétexte pour l'interview. Dans ces cas, on peut constater qu'il y a des différences par rapport aux figures précédemment identifiées - ou, du moins, occasion d'approfondir la réflexion à ce propos. Sans entrer dans le détail de l'analyse, une fois de plus, essayons de mieux préciser ce dont il est question. Dans ces introductions, le thème est fréquemment le chanteur lui-même. Celui-ci est souvent présenté (représenté) dans une temporalité chronologique (ou biographique), qui débute par des segments avec disjonction temporelle et se ferme avec conjonction temporelle (marquée, notamment, par l'occurrence explicite du déictique temporel agora /maintenant et par l'occurrence du présent de l'indicatif à valeur déictique). Même s'il y a des actions qui sont représentées, ce n'est pas l'agir qui est thématisé, mais l'histoire (de l'agir) de l'objet - en l'occurrence, le chanteur Janita Salomé. En même temps (souvent dans la même introduction), on retrouve des segments où le thème est repris du point de vue d'une stabilisation définitoire de l'ordre de l'exposé. Finalement, il faudra souligner les moments où le point de vue du journaliste (textualisateur) se fait entendre - c'est-à-dire où il fait entendre ses appréciations ou commentaires. Nous pourrons dire, pour l'ensemble, qu'il n'y a pas un travail de représentation discursive de l'agir, ou, en d'autres termes, qu'il n'y a pas, à proprement parler, de figures d'action. Il y a pourtant action, c'est-à-dire qu'il y a une dimension praxéologique liée à ce que le journaliste est en train de faire (l'éloge du chanteur, dans la plupart des cas), en parlant de son thème (le chanteur).

C'est en fonction des synthèses précédentes que nous pourrons mieux cerner l'intérêt de la présente démarche, en ce qui concerne les pratiques textuelles en contexte scolaire/académique. Le troisième groupe de textes avec lequel nous avons travaillé est constitué par des «exercices en contexte académique portugais $\left(1^{\text {rc }}\right.$ année, $1^{\text {cr }}$ cycle en langues et littératures/traduction). À la fin de la troisième session du cours (optionnel) nommé «Expression et Argumentation», les élèves ont dû faire un devoir obéissant à la consigne suivante: Si vous devez expliquer à quelqu'un ce que c'est le cours d' Expression et Argumentation ", que diriez-vous? Rédigez un texte court (maximum 10 lignes), en disant de quoi s'agit-il, dans ce cours. Un nombre significatif des textes reproduit, de façon plus ou moins proche, des passages du cours, tels que le professeur les a proférés ou montrés (passages que les élèves ont pu facilement récupérer par les présentations power point disponibles à travers la plateforme Moodle). La confrontation des deux 


\section{DOSSIFR}

productions (production du prof/production de l'élève collée à celle du prof) met en évidence que l'usage du discours théorique ne constitue pas une vraie garantie de qualité académique. En fait, les copies qui se sont détachées sont plutôt celles qui arrivent à établir un point de vue particulier sur le thème (le cours).

C'est le cas de cet élève qui raconte un épisode de sa vie pour le mettre en rapport avec le fait d'avoir choisi le cours (même si, à la fin, il y a le collage un peu brusque d'une sorte de définition) : «Em agosto, pleno verão, com alguma curiosidade, comecei a ler um livro (...). Foi por isso que me inscrevi nesta disciplina. Para entender o que faz afinal um bom discurso argumentativo. Para compreender o que tem vindo a ser debatido nas aulas. Argumentar é expor um conjunto de razões a serem defensoras de uma conclusão. » / Au mois d'août, en plein été, avec une certaine curiosité, j'ai commencé à lire un livre (...). C'est pourquoi j'ai choisi de m'inscrire à ce cours. Pour comprendre comment on fait, finalement, un bon discours argumentatif. Pour comprendre ce qu'on vient de discuter pendant les cours. Argumenter, c'est exposer un ensemble de raisons qui plaideront pour une conclusion.

C'est le cas de cet autre élève qui réfléchit sur l'expérience quotidienne d'expression et d'argumentation pour conclure sur l'importance du cours : «Argumentar é um processo importante que faz parte do nosso dia-a-dia. (...) Sem esta capacidade de exprimirmos os nossos pensamentos aos outros, ficaríamos limitados e fechados às nossas próprias ideias, e é assim que esta disciplina se torna importante na medida em que irá desenvolver as nossas capacidades de argumentar (...).»/ Argumenter, c'est un processus important qui fait partie de notre quotidien. (...) Si nous n'avions pas cette capacité d'exprimer aux autres nos pensées, nous serions limités et fermés dans nos propres idées, et c'est pourquoi ce cours devient important, dans la mesure où il développera nos capacités d'argumenter.

C'est encore le cas, très curieux, de cet élève qui saisit le thème vraiment, du point de vue de l'action future : «Estudaremos os mecanismos da língua verbal (...); e analisaremos aquilo que é linguisticamente apresentado como argumento »/ Nous étudierons les mécanismes du langage verbal (...); et nous analyserons ce qui est linguistiquement présenté en tant que argument").

Les différentes possibilités attestées par ces exemples suggèrent que la réussite des pratiques textuelles scolaires/académiques ne passe pas, en général, par une représentation discursive de l'agir mais plutôt par une sorte de compréhension praxéologique des enjeux associés à la tache dont il est question. En fait, cela nous force à conclure en gardant ouverte cette voie de réflexion: l'aisance de figuration thématique, ne serait-elle pas une condition majeure du développement de la personne (adulte) et, de façon particulière, du développement des performances académiques?

\section{RÉFÉRENCES BIBLIOGRAPHIQUES}

Maria ANTONIA COUTINHO (acoutinho@ fcsh.unl.pt) FCSH-UNL, CLUNL, Universidade Nova de Lisboa,

ADAM, J.-M. (2011). La linguistique textuelle ( $3^{\mathrm{c}}$ édition). Paris: Armand Colin

BRONCKART, J.-P. (2008). Genre de textes, types de discours et "degrés" de langue. Texto!, XIII(1), [En ligne]. URL: http://www.revuetexto. net/index.php?id=8.

BRONCKART, J.-P. \& E. BUlEA (2006). La dynamique de l'agir dans la dynamique langagière. In J.-M. Barbier \& M. Durand (eds). Sujet, activité, environnement : approches transverses. Paris : PUF, $105-$ 134

BulEA, E. (2007). Le rôle de l'activité langagière dans l'analyse des pratiques à visée formative. Thèse de doctorat. Université de Genève

BulEA, E. (2009). Types de discours et interprétation de l'agir: le potentiel développemental des figures d'action. Estudos linguísticos/Linguistic Studies 3, 135-152

TeiXeira, C. \& Coutinho, M. A. (2011). Ser e estar em figuras de acção nos textos sobre o vinho, in M.T. Brocardo (org.). Cadernos WGT - Ser \& Estar. Balanços e Perspectivas. Lisboa : CLUNL, $31-41$. 